ApJ, in press

\title{
Monitoring the Bi-Directional Relativistic Jets of the Radio Galaxy 1946+708
}

\author{
G. B. Taylor ${ }^{1}$, P. Charlot ${ }^{2,3}$, R. C. Vermeulen ${ }^{4}$, and N. Pradel ${ }^{4,5}$ \\ gbtaylor@unm.edu
}

\begin{abstract}
We report on a multi-frequency, multi-epoch campaign of Very Long Baseline Interferometry observations of the radio galaxy 1946+708 using the VLBA and a Global VLBI array. From these high-resolution observations we deduce the kinematic age of the radio source to be $\sim 4000$ years, comparable with the ages of other Compact Symmetric Objects (CSOs). Ejections of pairs of jet components appears to take place on time scales of 10 years and these components in the jet travel outward at intrinsic velocities between 0.6 and 0.9 c. From the constraint that jet components cannot have intrinsic velocities faster than light, we derive $H_{0}>57 \mathrm{~km} \mathrm{~s}^{-1} \mathrm{Mpc}^{-1}$ from the fastest pair of components launched from the core. We provide strong evidence for the ejection of a new pair of components in $\sim 1997$. From the trajectories of the jet components we deduce that the jet is most likely to be helically confined, rather than purely ballistic in nature.
\end{abstract}

Subject headings: cosmology:distance scale — galaxies: active — galaxies: individual $(1946+708)$ - galaxies: jets — radio continuum: galaxies

\section{Introduction}

Compact symmetric objects (CSOs) are a family of extragalactic radio sources comprising roughly $3 \%$ of flux-limited samples selected at high frequencies (Taylor, Readhead, \& Pearson

\footnotetext{
${ }^{1}$ Department of Physics and Astronomy, University of New Mexico, Albuquerque NM, 87131, USA; gbtaylor@unm.edu. Greg Taylor is also an Adjunct Astronomer at the National Radio Astronomy Observatory.

${ }^{2}$ Université de Bordeaux, Observatoire Aquitain des Sciences de l'Univers, 2 rue de l'Observatoire, BP 89, 33271 Floirac Cedex, France

${ }^{3}$ CNRS, Laboratoire d'Astrophysique de Bordeaux - UMR 5804, 2 rue de l'Observatoire, BP 89, 33271 Floirac Cedex, France

${ }^{4}$ Netherlands Foundation for Research in Astronomy (ASTRON), PO Box 2, 7990 AA Dwingeloo, The Netherlands

${ }^{5}$ National Astronomical Observatory of Japan, 2-21-1 Osawa, Mitaka, Tokyo, Japan
} 
1996; Peck \& Taylor 2001). Their defining characteristic is the presence of high luminosity radio components on both sides of a central engine on sub-kiloparsec scales with little or no extended emission present. CSOs typically exhibit terminal hotspots which move apart at subluminal speeds (Owsianik \& Conway 1998; Gugliucci et al. 2005). Jet components en route between the core and the hot spots where they terminate, appear to move faster, and superluminal speeds in the jets are seen in a few sources (Taylor, Readhead \& Pearson 2000). The jets can be similar or much brighter than the counterjets. CSOs on the whole exhibit weak radio variability, have low radio polarization and low core luminosities. Their hosts are in general elliptical galaxies (Readhead et al. 1996) though there are a few notable exceptions identified with Seyferts and quasars.

The general picture that has emerged is that CSOs are young radio galaxies, with ages between 100 and 10000 years. Since they are selected on the basis of relatively unbeamed hot-spots and lobes, their orientation on the sky is random. Orientation may still affect the presence of detectable linear polarization (Gugliucci et al. 2007), or absorption from atomic gas associated with the hypothesized gas and dust torus that surrounds the central engine and is thought to be oriented perpendicular to the jet axis (Peck et al. 2000; Pihlström et al. 2003). However, as Doppler boosting effects are less dominant, we have the added benefit of studying the emission from both sides of the nucleus.

CSOs provide a unique opportunity to determine the Hubble constant, as a direct measurement of the distance to an object can be obtained by observing angular motion in it, if the intrinsic linear velocity can be ascertained independently. Basically, one has the time derivative of a standard ruler, with the additional constraint that no intrinsic motion can be faster than light. From five epochs of observations of the CSO $1946+708$ at $5 \mathrm{GHz}$ Taylor \& Vermeulen (1997) placed a lower limit of $\mathrm{H}_{0}>37 \mathrm{~km} \mathrm{~s}^{-1} \mathrm{Mpc}^{-1}$. We explore what can be learned from continuing this analysis using more comprehensive observations. We also use this symmetric system, for which the geometry of the jets can be precisely determined, to probe the details of jet propagation.

Kinematic ages for the radio emission in CSOs can be obtained by measuring the separation speed between the hot spots over time and comparing this to the overall spatial separation (Owsianik \& Conway 1998; Gugliucci et al. 2005). At the moment, the CSO age distribution seems to be disproportionally stacked towards younger sources. However, there are a number of selection effects that need to be addressed before the meager data collected can be properly analyzed. Larger sources are over-resolved by VLBI observations so that measuring the expansion becomes difficult; more slowly expanding sources require longer time baselines to obtain age estimates. In this work we present observations of the CSO $1946+708$ spanning 16 years. It is of considerable interest to pursue this line of research to address the evolution of radio galaxies.

We assume $H_{0}=71 \mathrm{~km} \mathrm{~s}^{-1} \mathrm{Mpc}^{-1}$ and the standard cosmology so that $1^{\prime \prime}=1.835 \mathrm{kpc}$ and an angular velocity of $1 \mathrm{mas} / \mathrm{y}=5.98 \mathrm{c}$ at the redshift $(0.1008)$ of the radio galaxy $1946+708$ (Peck \& Taylor 2001). 


\section{Observations and Data Reduction}

The observations were carried out at $8.4 \mathrm{GHz}$ and $15.4 \mathrm{GHz}$ over the course of eight epochs, taken on 1995 March 22, 1996 July 07, 1998 February 06, 1999 July 11, 2001 May 17, and at 8.4 GHz only on 2003 Jan 24/2003 Feb 10, 2006 Feb 18, and 2008 Mar 9 (See Table 1). Observations in 1995, 1998, and 2001 were observed using all ten elements of the VLBA of the NRAO, and observations from 2003 on were observed using a global array including all ten elements of the VLBA, and five elements from the European VLBI Network (EVN) including the $100 \mathrm{~m}$ telescope at Effelsberg, the Westerbork phased array, Onsala, Medicina, and in 2003 only, the telescope at Noto. Due to problems at the St. Croix and North Liberty stations, epochs in 1996 and 1999 were performed using nine VLBA antennas each. Problems in 2003 prevented Effelsberg from participating. Both right and left circular polarizations were recorded for the first 5 epochs, while the last three where observed only in right circular polarization. All epochs employed 2 bit sampling across IF bandwidths of $8 \mathrm{MHz}$. The VLBA correlator produced 16 frequency channels across each IF during every $2 \mathrm{~s}$ integration. We also include in our analysis the $5 \mathrm{GHz}$ observations acquired between 1992 and 1995 by Taylor \& Vermeulen (1997), some of which are contemporaneous with the 8.4 and $15 \mathrm{GHz}$ observations reported here.

Parallactic angle effects resulting from the altitude-azimuth antenna mounts were removed using the AIPS task CLCOR. Amplitude calibration for each antenna was derived from measurements of antenna gain and system temperatures during each run. Delays between the stations' clocks were determined using the AIPS task FRING (Schwab \& Cotton 1983). Calibration was applied by splitting the multi-source data set immediately prior to preliminary editing, imaging, deconvolution and self-calibration in Difmap (Shepherd, Pearson, \& Taylor 1994, 1995). Multiple iterations of phase self-calibration and imaging were applied to each source before any attempt at amplitude self-calibration was made. The preliminary models developed in Difmap were subse-

quently applied in AIPS to make phase corrections, to determine the leakage terms between the RCP and LCP feeds and to correct for residual phase differences between polarizations. Final imaging and self-calibration were performed in Difmap.

Model fitting of Gaussian components to the self-calibrated visibility data was also performed in Difmap. The shapes of the components were fixed after fitting to the 1995 epoch; in the rest of the epochs each component was allowed only to move and to vary in flux density in order to fit the independently self-calibrated visibility data. Each frequency band $(5,8.4$ and $15.4 \mathrm{GHz})$ was modelfit independently in order to allow for differences in component shapes between frequencies. For our last three epochs from 2003 to 2008, all global $8.4 \mathrm{GHz}$ observations, an additional pair of components had to be fit on either side of the core (see $\$ 4.1$ ). Uncertainties in the sizes and positions for components were derived from signal-to-noise ratios and component sizes (Fomalont 1999). This assumes that parameters are not covariant, which should be the case for the strong, well-separated components that we present in this analysis of $1946+708$. 


\section{Results}

While one of the first objects to be confirmed as a bona-fide Compact Symmetric Object, $1946+708$ is in a few ways unusual for this class. The source is remarkably "rotation" symmetric (see Figures 1 and 2). Narrow, well collimated jets emerge from a compact, flat spectrum core and bend gradually before terminating in well defined hot spots. Faint "lobes" of emission are evident at $90^{\circ}$ angles to the jets. Distinct components in the jet are seen to move out from the core to the hot spots on both the Northern (jet) side and the Southern (counter-jet) side.

Our most uniform, sensitive, and highest resolution sequence of images is that at $8.4 \mathrm{GHz}$. The 8.4 GHz images cover the period from March 1995 to March 2008, and have noise levels ranging from $163 \mu \mathrm{Jy} /$ beam down to $38 \mu \mathrm{Jy} /$ beam (Table 1).

No polarized flux has been detected from $1946+708$ at either 8.4 or $15.4 \mathrm{GHz}$ in any epoch. Typical $2 \sigma$ limits on the linearly polarized flux density are $<100 \mu \mathrm{Jy}$ at $8.4 \mathrm{GHz}$ and $<170 \mu \mathrm{Jy}$ at $15.4 \mathrm{GHz}$.

\section{Discussion}

\subsection{A New Set of Jet Components}

This source is quite steady in the production of strong and distinct jet components. In addition to the previously studied pairs N2/S2 and N5/S5, we report here on the discovery of a new set of components which we name N6/S6 (Fig. 2). Like the previous sets, these new components appear to have been launched simultaneously as a pair, and appear to be moving away from the core in opposite directions.

The angular separation between the new pair (N6 and S6) observed in our 2008 epoch is 1.288 mas. The apparent proper motion between N6 and S6 and the core is difficult to determine owing to possible blending of these components with the core, but the total separation can be compared with the 2006 epoch in which it was 1.045 mas. The total apparent expansion speed between the pair over the past two years is thus 0.238 mas/y or $0.72 \mathrm{c}$. This puts the ejection age for the new

pair at 1997.3. Indeed evidence of the new component set can be seen in the 2003 epoch, and to a lesser extent in 2001. The sum of the core flux density and the flux density of the new components N6 and S6 during this period (Fig. 3) was rising steadily, and only seems to be declining later on, as the new components move well away from the core.

\subsection{Kinematics in the jets of $1946+708$}

In Fig. 4 we plot the positions of the 4 strongest jet components (N2, N5, S5 and S2) derived from modelfitting elliptical gaussians to the visibility data at $8.4 \mathrm{GHz}$. The well-tracked jet 
components close to the core, N5 and S5, appear to move out with the highest apparent velocity (Table 2 and Fig. 5; and see also the Movie6), with N5 being the fastest at an apparent velocity of $1.088 \mathrm{c}$, and S5 being about one third that in very nearly the opposite direction. Note that these are apparent velocities projected in the plane of the sky, as opposed to intrinsic, three-dimensional velocities. The more distant pair of components (N2/S2) appear to be moving at roughly half the apparent velocity of the inner (N5/S5) pair. From this fact one might be tempted to assert that the jet starts out fast and decelerates with time. If this was the case, and the deceleration is uniform, then we would expect to see the N5/S5 pair slowing down, but this is not observed. Even after 12 years of monitoring and a distance covered corresponding to $10 \%$ of the length of the jet, there is no compelling evidence for slowing of any of the components. The $\chi^{2}$ values indicate reasonably good fits to a straight line for all components (the $\chi^{2}$ values may be systematically better in declination than in right ascension owing to the north-south orientation of most of the components). If current velocities remain unchanged then the N5/S5 pair will catch up with the $\mathrm{N} 2 / \mathrm{S} 2$ pair in $\sim 75$ years, close to the time that they enter the hot spots. Alternatively, it could be that there is a sudden deceleration of the jet components at a distance from the core between 3.5 and 9 mas (on the northern side). This gap is defined by the minimum distance of 9 mas for N2 in 1995 and the maximum distance for N5 observed in 2008. This is in the region where the jet bends the most $\left(\sim 40^{\circ}\right)$ towards the northern hot spot, so a deceleration region should not be ruled out prematurely. Considering the newest pair of components, N6/S6, their total apparent separation speed is $0.72 \mathrm{c}$, more like that of the N2/S2 pair $(0.63 \mathrm{c})$, than the N5/S5 pair $(1.4 \mathrm{c})$. Observations over the next $\sim 5$ years should settle the issue of whether components emerge with their own, intrinsic set of velocities, or if they partake in some fixed pattern of acceleration and deceleration. If there is a fixed, repeating pattern, then it might be possible to relate it to a helical jet model. In a helical model, the intrinsic velocity could be constant while the orientation changes in time to produce the observed variations in the projected apparent velocities. Such a model naturally reproduces the rotational symmetry of $1946+708$ and has been found to explain observations over many years of the galactic jet in SS 433 (Roberts et al. 2008, Hjellming \& Johnston 1981).

The path of the inner jet components of 1946+708 (N5 and S5) both appear fairly straight to within the errors in the measurements. There is a suggestion of a bend in the trajectory of N5 after the 2006 epoch (epoch 9 in Fig. 4), but this relies almost entirely on the position at the 2008 epoch. For the more distant northern component (N2) there are kinks in the apparent motion near epochs 1994, 2001 and 2006 (epochs 2, 7 and 9 in Fig. 4). There also appears to be an offset in the $15 \mathrm{GHz}$ positions (see Fig. 4). This suggests a flatter spectrum on the eastern side of this component. A similar offset is suggested by the multi-frequency trajectory plot of $\mathrm{S} 2$ based on the 5 and $8 \mathrm{GHz}$ observations, but since the motions are smaller and the component is weaker it is difficult to be sure if the spectral gradient is real. A kink is observed in component S2 near the 2003 epoch (epoch 8 in Fig. 4).

\footnotetext{
${ }^{6}$ The Movie is available from http://www.phys.unm.edu/ gbtaylor/
} 
In well studied core-jet sources, such as 3C 345 (Zensus et al. 1995, Unwin et al. 1997), jet components are found to travel along curved trajectories and to change their speeds. This acceleration has been taken as justification for magnetically driven jets, as opposed to purely hydrodynamical structures (Vlakhis \& Konigl 2004). By analogy with these core-jet sources, we draw a similar conclusion that the jets of $1946+708$ are likely to be magnetically driven. Note, however, that intrinsic velocities of jet components in core-jet AGNs are typically in the range $\gamma \sim 3-10$, considerably larger than we observe in the CSO 1946+708 of $1.3-2.3$ (see below).

Even before the core of $1946+708$ was identified based on $15 \mathrm{GHz}$ observations, the first two epochs at $5 \mathrm{GHz}$ suggested its location by exhibiting bi-directional motions away from the center of the source. Given these motions and the symmetry of the source (Figs. 1 and 2), it is reasonable to assert that N5 and S5 were ejected at the same time, and likewise N2 and S2. Indeed, we are fortunate enough to witness the ejection of N6 and S6 in 1997 as discussed above. Sensitive observations at $8.4 \mathrm{GHz}$ (see Fig. 2), reveal that there is continuous emission from the core out to both hot spots. The jet is probably not made up of discrete blobs that can be well described by the elliptical Gaussian components that we identify and modelfit. Rather the jet appears to be a continuous flow, with features of enhanced emission (shocks?) that propagate down the jet.

Under the assumption that components are ejected in pairs, and that all differences are due to Doppler boosting and light travel time effects, we can use the observed apparent velocity ratios and differences to solve for the intrinsic component velocity and orientation. For simultaneously ejected components moving in opposite directions at an angle $\theta$ to the line of sight at an intrinsic velocity $\beta$, it follows directly from the light travel time difference that the ratio of apparent projected distances from the origin ( $d_{\mathrm{a}}$ for the approaching side, $d_{\mathrm{r}}$ for the receding side) as well as the ratio of apparent motions (approaching: $\mu_{\mathrm{a}}$, receding: $\mu_{\mathrm{r}}$ ) is given at any time by (Taylor \& Vermeulen 1997):

$$
\frac{\mu_{\mathrm{a}}}{\mu_{\mathrm{r}}}=\frac{d_{\mathrm{a}}}{d_{\mathrm{r}}}=\left(\frac{1+\beta \cos \theta}{1-\beta \cos \theta}\right) .
$$

Our other important constraint on the two parameters $\beta$ and $\theta$ can be obtained from the separation rate $\mu_{\text {sep }}=\left|\mu_{\mathrm{a}}\right|+\left|\mu_{\mathrm{r}}\right|$, which, unlike $\mu_{\mathrm{a}} / \mu_{\mathrm{r}}$, is not subject to the uncertainty in the reference point. From geometry and the conversion of angular to linear velocity we have:

$$
v_{\text {sep }}=\mu_{\text {sep }} D_{a}(1+z)=\frac{2 \beta c \sin \theta}{\left(1-\beta^{2} \cos ^{2} \theta\right)},
$$

where $v_{\text {sep }}$ is the projected separation velocity, $D_{a}$ is the angular size distance to the source, and $z$ is the redshift. Note that Eq. 2 has a distance dependence, while Eq. 1 does not, so we can solve the system jointly for the distance and hence the Hubble constant.

For the N5/S5 pair we find an apparent velocity ratio $\mu_{\mathrm{a}} / \mu_{\mathrm{r}}$ of $3.50 \pm 0.44$. This leads to $\beta \cos \theta=0.56 \pm 0.04$. At the same time, the apparent separation speed of the N5/S5 pair is 1.40 \pm 0.01 c. The two relations above are shown graphically in Fig. 6 (bottom panel). Assuming a standard cosmology and $\mathrm{H}_{0}=71 \mathrm{~km} \mathrm{~s}^{-1} \mathrm{Mpc}^{-1}$ we find a common solution for an intrinsic velocity 
of $0.88 \pm 0.03 \mathrm{c}$, at an inclination of $50 \pm 5^{\circ}$. A similar analysis for the N2/S2 pair (top panel of Fig. 6), yields in intrinsic velocity of $0.57 \pm 0.11 \mathrm{c}$ and with an inclination of $36 \pm 10^{\circ}$. The intrinsic velocity therefore changes from 0.9 c for N5/S5 to 0.6 c for N2/S2 (a change of $2.7 \sigma$ ). There is no single intrinsic velocity that can fit the observations for both pairs, and can be used to directly measure the Hubble constant. We can, however, place a lower limit on the Hubble constant based on the fact that the intrinsic velocity must be less than the speed of light. We find that $\mathrm{H}_{0}>57$ $\mathrm{km} \mathrm{s}^{-1} \mathrm{Mpc}^{-1}$ in order to achieve a valid solution for the N5/S5 pair, and the weaker constraint that $\mathrm{H}_{0}>28 \mathrm{~km} \mathrm{~s}^{-1} \mathrm{Mpc}^{-1}$ in order to achieve a valid solution for the N2/S2 pair. Since the velocities of N2/S2 differ from those of N5/S5 anyway, we see no reason why the latter should be particularly close to $\mathrm{c}$, so for the rest of this discussion we adopt $\mathrm{H}_{0}=71 \mathrm{~km} \mathrm{~s}^{-1} \mathrm{Mpc}^{-1}$.

The solutions discussed above using equations 1 and 2 are based on assuming that the components in a pair, as observed, are oppositely directed and equally fast. We derive angles near $45^{\circ}$; the line of sight depth difference between the components in a pair would therefore be an appreciable fraction of their total distance along the connecting line through the core, and roughly equal to the distance projected onto the plane of the sky. We derive component speeds that are an appreciable fraction of the speed of light. In combination, the speed and angle solutions imply that the receding component, due to a significant light travel time difference, should be observed at a rather younger age (25\%-75\%) than its approaching counterpart, and at a correspondingly smaller projected distance from the core. However, the observed arm length ratios $\left(d_{a} / d_{r}\right)$ are lower by a factor of about 2 compared to the apparent velocity ratios (1.33 vs. 2.76 for N2/S2; and 1.80 vs. 3.50 for N5/S5). This requires that currently the light travel time differences between the approaching and receding components are less significant with respect to the ages of the components than our simple speed and angle solutions suggest. The arm length ratios depend on the entire history of motion of the components, and the greater degree of symmetry in arm lengths seems to imply that for a significant part of their lifetime the components were slower and/or moving more along the plane of the sky than they are now. A fully self-consistent solution therefore will incorporate a model for the true core location and for this evolution in velocity, since, inevitably, it implies that the receding component is seen at a younger age and therefore with a different velocity than its counterpart. This leads to an extension of equations 1 and 2, into a set where the arm length ratio is directly incorporated; helical jet models may provide a framework for this extension. However, it is beyond the scope of this paper. To properly verify such a model in the case of $1946+708$ will require observations spanning several decades. Nevertheless, we believe the velocities we have derived, while not fully self-consistent, are indicative of the fact that the jets of this source contain features that move with speeds that are an appreciable fraction of the speed of light, and certainly much faster than the advance speeds of the hot spots. Furthermore, the differences in angle (by at least 15 degrees) as well as in speed (by at least 50\%) between the N2/S2 and N5/S5 pairs are good indications of the kinds of changes in component velocity that are evidently occurring in and along the jets. Both the discrepant arm length ratios and the observed bending in projection of the overall jets on the plane of the sky provide interesting constraints. 


\subsection{Flux Density Evolution}

The time evolution of the flux densities of the N2/S2 and N5/S5 pair is shown in Fig. 7. The N2 component has shown a steady rise by $\sim 20 \%$ over 13 years of monitoring at $8.4 \mathrm{GHz}$. During that same time S2 has only slightly decreased. Meanwhile both N5 and S5 have been declining though that appears to have leveled off starting in 2006. The amount of the decline was $75 \%$ for N5 and 57\% for S5. We are currently in the unbalanced situation where S5 appears to be brighter than N5, which would at first sight seem contrary to the expectations from Doppler boosting. However, we have to keep in mind that the light from S5 is delayed compared to the light that we see from N5 (by 27 years for the 2008 epoch according to the geometry derived above) so that the history of variability must be taken into account when attempting to interpret flux density ratios between component pairs. A more detailed analysis of the flux density ratios, taking into account the time variability, should eventually be possible. Even so, it may not be possible to explain the very significant changes in flux density shown in Fig. 7. given the stability of the component velocities (see Fig. 5). Local circumstances (e.g., variations in magnetic field strength or particle populations), and possible interactions of the jet components with their environments, may influence the synchrotron emissivity of the jet components.

\subsection{Non-ballistic Motions}

The curvature in the jets of $1946+708$ (Fig. 1) could be explained by either (1) ballistic motion from a precessing nucleus; or (2) helical confinement of the components. In the first case we would expect components to travel in straight lines at constant apparent velocities. In the latter case we would expect that components might travel along helical trajectories at apparent velocities that changed in time. Although there is no strong evidence yet for deviations from constant apparent velocities, there do appear to be some wiggles or kinks in the jets, and in particular if one considers also the earliest $5 \mathrm{GHz}$ measurement (C1 in Fig. 4), then there appears to have been a change in direction of several degrees. This favors the second explanation of a helically confined jet. Likewise, the presence of continuous emission along the jet, well confined, even if traveling at different intrinsic velocities (N5 vs N2), supports the notional model of an intrinsically helical jet.

\subsection{The Advance Speed of the Hot Spots}

From the modelfit analysis it is difficult to ascertain the expansion of the source. Certainly it is clear that the motion is much slower than the jet components. Unfortunately the southern hot spot

is fairly faint and diffuse, so is correspondingly less amenable to modelfitting than the compact jet components. The northern hot spot does have a bright and compact feature, and one can measure with considerable accuracy its position relative to the core. The observed motion is $<0.01 \mathrm{mas} / \mathrm{y}$, at the level that one has to be concerned about possible motions of the core component due to the 
ejection of the N6/S6 pair. Since the modelfit results are referenced to the core component, any apparent proper motion of the core translates into the addition of a systematic apparent velocity to all components.

An alternative approach is to compute a difference map between two images, well separated in time. Apparent motions then show up as alternating positive and negative structures. Fig. 8 shows such an image constructed by differencing the images from the 1996 and 2008 epochs - both of which are of very high quality and low noise, and were convolved with the same restoring beam. Lighter regions in Fig. 8 indicate where the source was brighter in 2008. In Fig. 8 one can see a relatively clear signature of motion away from the core of the southern hot spot, which appears as a positive (dark) structure (closer to the core) and a negative (whiter) structure (further from the core) in the difference image. The more rapidly moving jet components show similar structures. From measuring the advance of the edge of the southern hot spot we estimate this apparent motion to be $0.008 \pm 0.002 \mathrm{mas} / \mathrm{y}$. Since the apparent motion measured is relative to the northern hot spot, this is the overall apparent expansion rate of the source, and the apparent motion of the southern hot spot would presumably be half this value, or $0.004 \mathrm{mas} / \mathrm{y}$, corresponding to a projected intrinsic velocity of $0.024 \mathrm{c}(7000 \mathrm{~km} / \mathrm{s})$.

Based on the measurement of the overall expansion rate, we can derive a kinematic age for 1946+708 assuming a constant expansion rate. The velocity measurement derived above yields a kinematic age of $4000 \pm 1000$ years. This is on the long end of CSO ages measured to date (Gugliucci et al. 2005), which have been measured between 100 and 3000 years, though the statistics are admittedly still poor. Furthermore, there is a selection effect that slow expansions take longer to measure.

The ratio of 15:1 for the northern to southern hot spot flux densities (derived from modelfitting), is difficult to explain in terms of Doppler boosting given the very low apparent velocities measured for the hot spots. A more likely explanation is that the interstellar medium may be enhanced on the northern side, consistent with the observation of greater HI opacity to the North (Peck \& Taylor 2001). The arm length ratio between northern and southern hot spots is close to parity, 0.94:1, with the northern hot spot being a little closer to the core. In the case of Doppler boosting we would expect the northern hot spot to be further away from the core, thus we favor a denser medium on the northern side to be responsible for the difference in flux densities and arm length ratios between the two hot spots.

\subsection{Depolarization by a Faraday Screen}

The low observed polarization (less than $0.4 \%$ for the core, less than $\sim 0.3 \%$ for the jet components, and less than $0.1 \%$ for the northern hot spot at $8.4 \mathrm{GHz}$ in 1996), can be explained by Faraday depolarization due to ionized gas and a magnetic field tangled on scales smaller than the angular resolution of the observations. This situation could naturally arise due to magnetic fields 
and free electrons associated with the accretion disk. Detection of polarization has only been found in a few CSOs to date (Gugliucci et al. 2007). Those few incidents of detected polarization occur in the approaching jets of CSOs which are more asymmetric and core-dominated than typical CSOs, probably indicating a smaller angle to the line-of-sight, and therefore a more shallow Faraday screen.

\section{Conclusions}

After a detailed, multi-frequency, multi-epoch study of the Compact Symmetric Object 1946+708, we find the kinematic age of the extant radio emission to be $\sim 4000$ years. On timescales of $\sim 10$ years, outbursts occur producing symmetric components that emerge from the core and travel at intrinsic speeds between 0.6 and $0.9 \mathrm{c}$ towards the hot spots. Some of the individual jet components are observed to move along slightly bent, or kinked paths, but no components have yet been observed to change their speed. The jet components in general appear to be well confined, and to lie within the overall "S-symmetric" shape of the jets. We suggest that the jets are helically confined, rather than ballistic in nature. No linear polarization from the jets or hot-spots is detected down to quite low levels (0.1 to $0.4 \%$ ), consistent with observations of CSOs in general.

Future observations over the next decade should allow for a detailed analysis of the newly ejected component pair N6/S6. Together with continued observations of N2/S2 and N5/S5 it should be possible to measure accelerations in the jet components, and to tell if each component pair has an intrinsic velocity that is established upon ejection. A more sophisticated analysis, taking into account the flux history of the jet components, could provide a more stringent test of Doppler boosting in $1946+708$ and thereby ascertain the extent to which interactions with the environment are important.

We thank H. Smith for help with the data reduction of the 1998 and 1999 epochs. G.B. Taylor gratefully acknowledges the University of Bordeaux for hosting a visit during which much of this work was undertaken. This work has benefited from research funding from the European Community's sixth Framework Programme under RadioNet R113CT 2003 5058187. The National Radio Astronomy Observatory is operated by Associated Universities, Inc., under cooperative agreement with the National Science Foundation. The European VLBI Network is a joint facility of European, Chinese, South African and other radio astronomy institutes funded by their national research councils. 


\section{REFERENCES}

Gugliucci, N.E., Taylor, G.B., Peck, A.B., \& Giroletti, M. 2005 ApJ, 622, 136

Gugliucci, N.E., Taylor, G.B., Peck, A.B., \& Giroletti, M. 2007 ApJ, 661, 78

Hjellming, R. M., \& Johnston, K. J. 1981, ApJ, 246, L141

Fomalont, E. B. 1999, Synthesis Imaging in Radio Astronomy II, 180, 301

Owsianik, I. \& Conway, J. E. 1998, A\&A, 337, 69

Peck, A. B., Taylor, G. B., Fassnacht, C. .D., Readhead, A. C. S., \& Vermeulen, R. C. 2000, ApJ, 534,104

Peck, A.B., \& Taylor, G.B. 2001, ApJL, 554, L147

Pihlström, Y. M., Conway, J. E., \& Vermeulen, R. C. 2003, A\&A, 404, 871

Readhead, A. C. S., Taylor, G. B., Xu, W., Pearson, T. J., Wilkinson, P. N., \& Polatidis, A. G. 1996, ApJ, 460, 612

Roberts, D. H., Wardle, J. F. C., Lipnick, S. L., Selesnick, P. L., \& Slutsky, S. 2008, ApJ, 676, 584

Schwab, F. R., \& Cotton, W. D. 1983, AJ, 88, 688

Shepherd, M. C., Pearson, T. J., \& Taylor, G. B. 1994, BAAS, 26, 987

Shepherd, M. C., Pearson, T. J., \& Taylor, G. B. 1995, BAAS, 27, 903

Taylor, G. B., Vermeulen, R. C., Pearson, T. J., Readhead, A. C. S., Henstock, D. R., Browne, I. W. A., \& Wilkinson, P. N. 1994, ApJS, 95, 345

Taylor, G. B., Readhead, A. C. S., \& Pearson, T. J. 1996, ApJ, 463, 95

Taylor, G. B., \& Vermeulen, R. C. 1997, ApJL, 485, L9

Taylor, G. B., Marr, J. M., Readhead, A. C. S., \& Pearson, T. J. 2000, ApJ, 541, 112

Unwin, S. C. Wehrle, A. E., Lobanov, A. P., Zensus, J. A., Madejski, G. M., Aller, M. F., \& Aller, H. D. 1997, ApJ, 480, 596

Vlahakis, N. \& Konigl, A. 2004, ApJ, 605, 656

Zensus, J. A., Cohen, M. H., \& Unwin, S. C. 1995, ApJ, 443, 35 


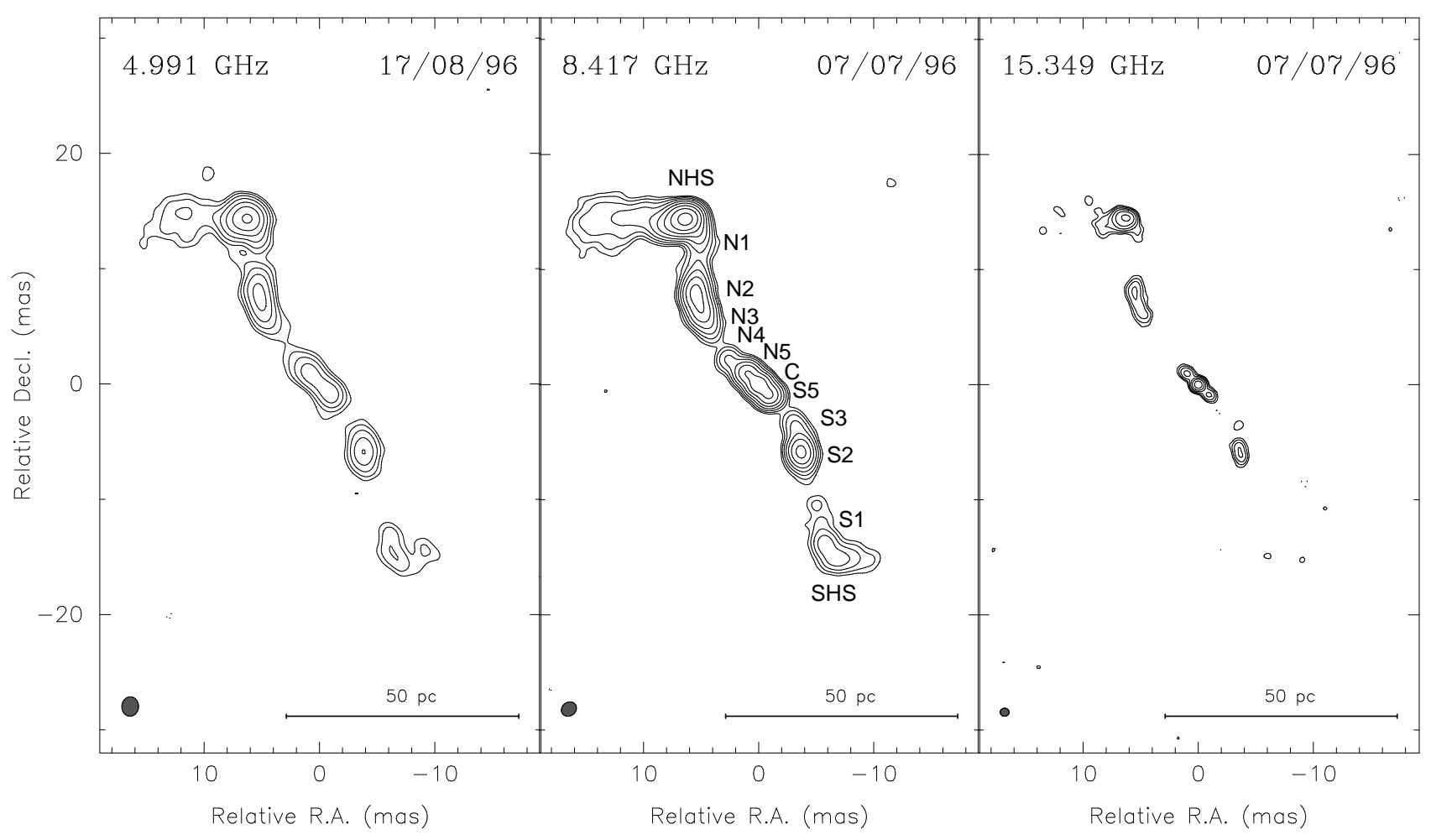

Fig. 1. - Nearly contemporaneous VLBA observations of $1946+708$ at 5,8 and 15 GHz. The synthesized beam is drawn in the lower left-hand corner of each plot and has dimensions: $1.68 \times$ 1.45 mas in position angle $-3.4^{\circ}$ at $5 \mathrm{GHz} ; 1.37 \times 1.18$ mas in position angle $-55^{\circ}$ at $8 \mathrm{GHz}$; and $0.78 \times 0.7$ mas in position angle $90^{\circ}$ at $15 \mathrm{GHz}$. Contours are drawn logarithmically at factor 2 intervals with the first contour at 2, 0.25, and $0.75 \mathrm{mJy} /$ beam at 5, 8 and $15 \mathrm{GHz}$ respectively. The components labeled NHS and SHS in the $8 \mathrm{GHz}$ image are the northern and southern hot spots respectively. The component labeled "C" we identify as the core. 


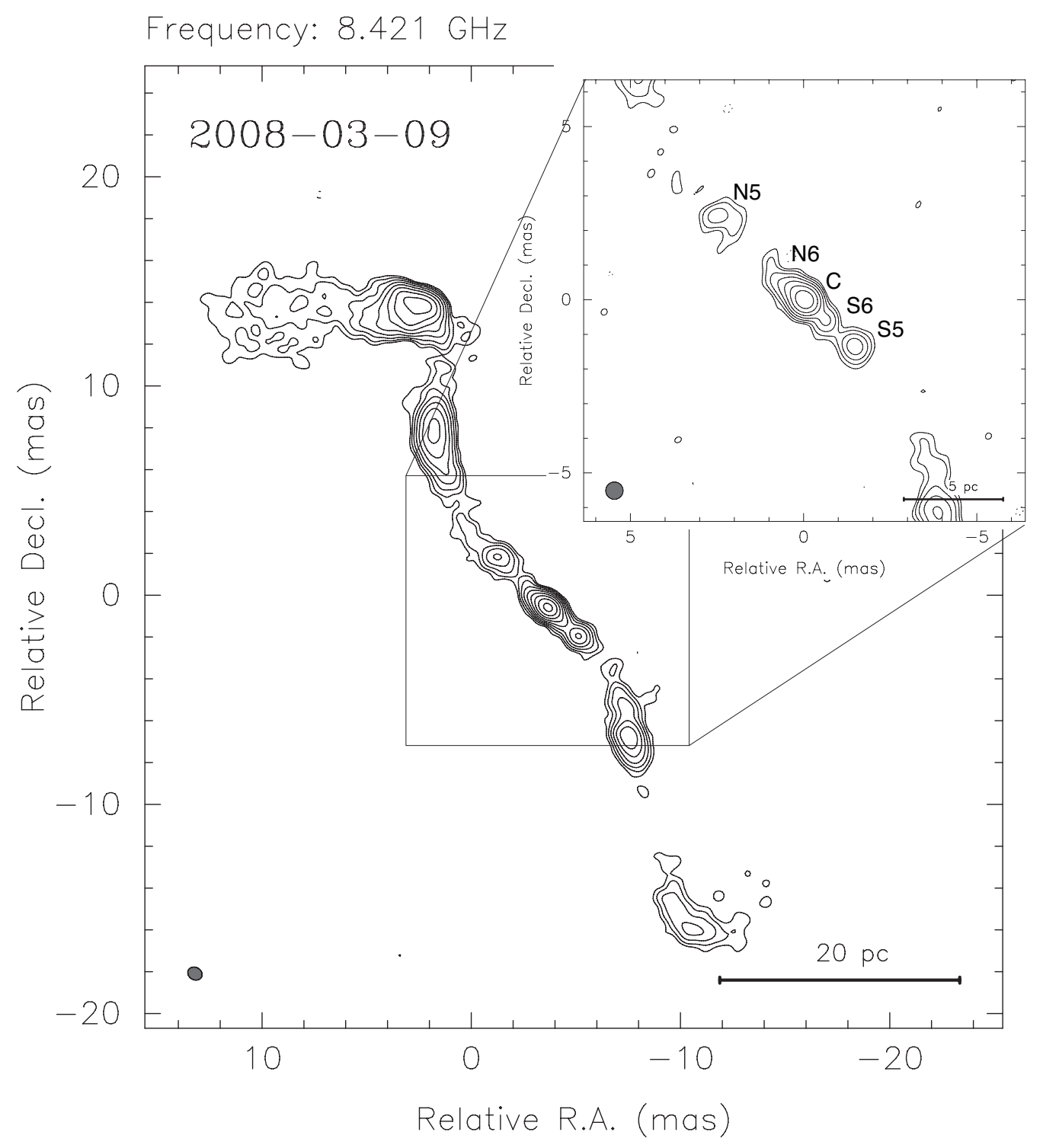

Fig. 2. - The naturally weighted $8.4 \mathrm{GHz}$ image from the 2008 epoch with a magnified view of the central region. The central region has full resolution and is uniformly weighted. The synthesized beam is drawn in the lower left-hand corner of each plot and has dimensions: $0.71 \times 0.59$ mas at position angle $62^{\circ}$ for the entire image and 0.5 mas for the inset image. Contours are drawn logarithmically at factor 2 intervals with the first contour at $0.2 \mathrm{mJy} /$ beam for the entire image and at $0.5 \mathrm{mJy} /$ beam for the inset. A new pair of components is visible just north and just south of the core. 


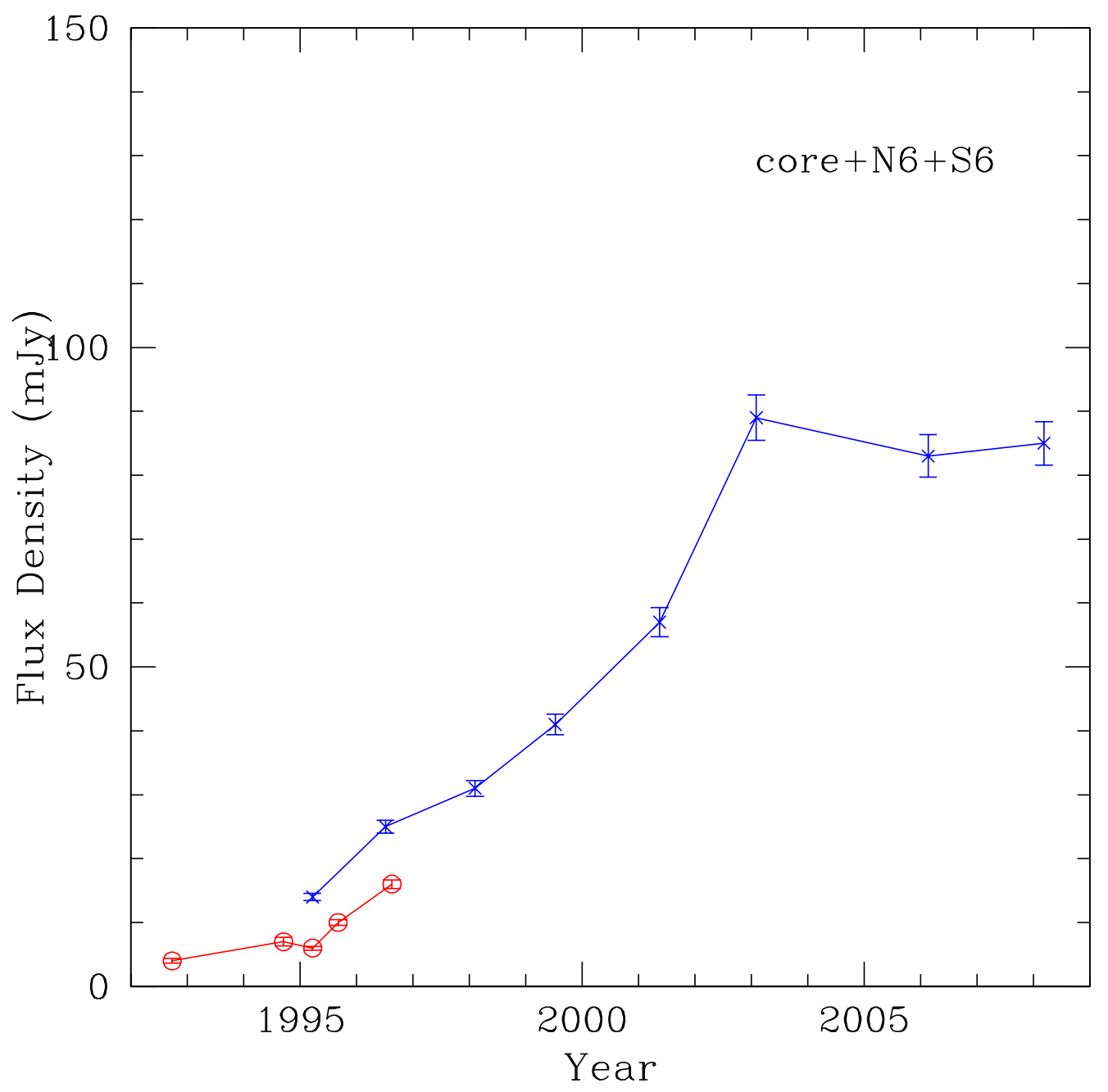

Fig. 3. - The flux densities of the core component between 1992 and 2008 as derived from Gaussian modelfitting. The discontinuity is the result of the first five monitoring epochs being at $5 \mathrm{GHz}$ (red), while the last eight epochs have used $8.4 \mathrm{GHz}$ (blue). 

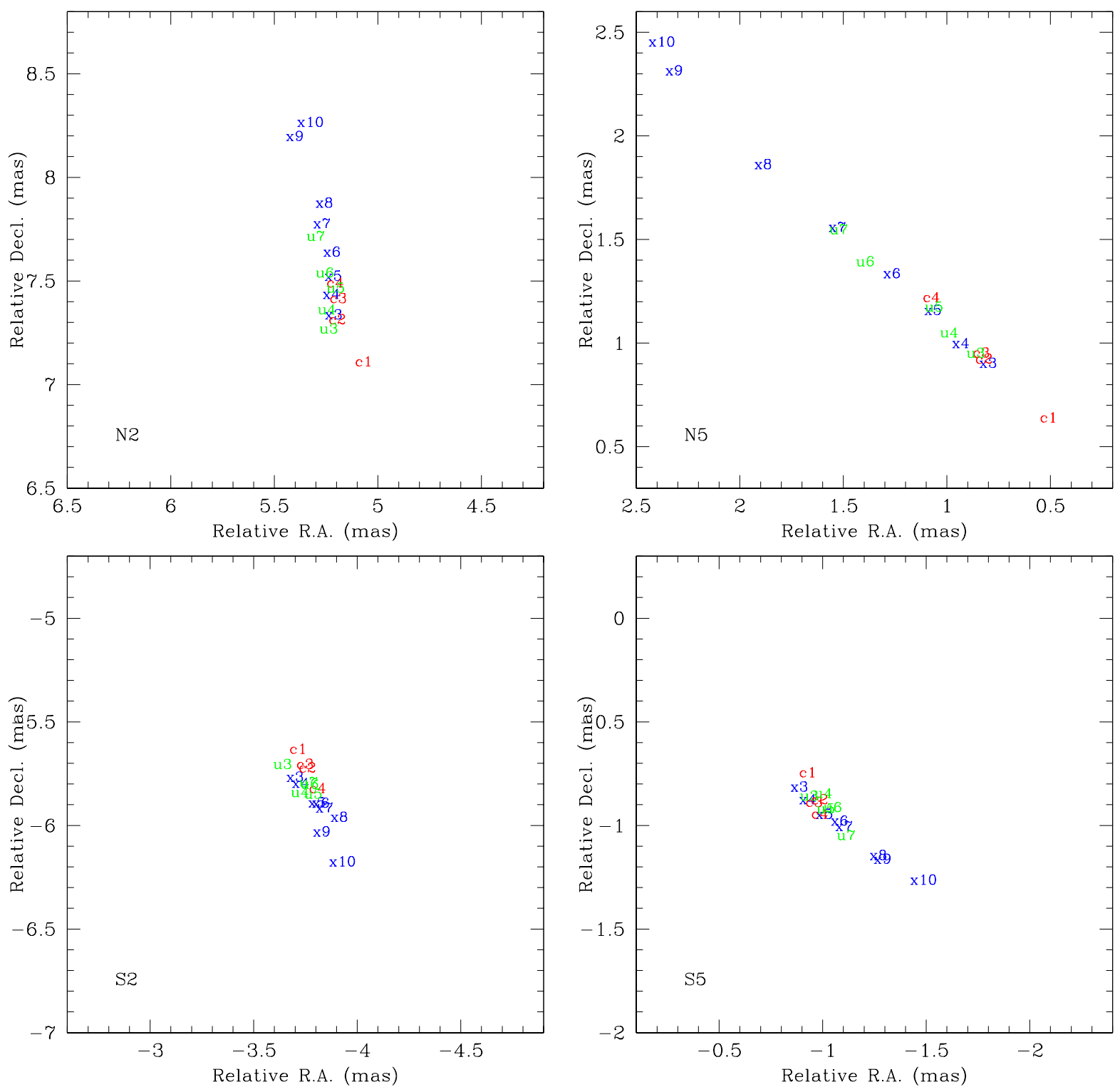

Fig. 4. - The path of the 4 paired jet components (N2/S2 and N5/S5) between 1992 and 2008 as derived from Gaussian modelfitting. Letters and colors designate the frequency band with red "c" for $5 \mathrm{GHz}$, blue "x" for $8.4 \mathrm{GHz}$, and green "u" for $15 \mathrm{GHz}$. The numbers correspond to the position of the component at epochs $1=1992$ Sep; $2=1994$ Sep; $3=1995$ Mar or Sep; $4=1996$ Jul or Aug; $5=1998$ Feb; $6=1999$ Jul; $7=2001$ May; $8=2003$ Feb; $9=2006$ Feb; $10=2008$ Mar. 

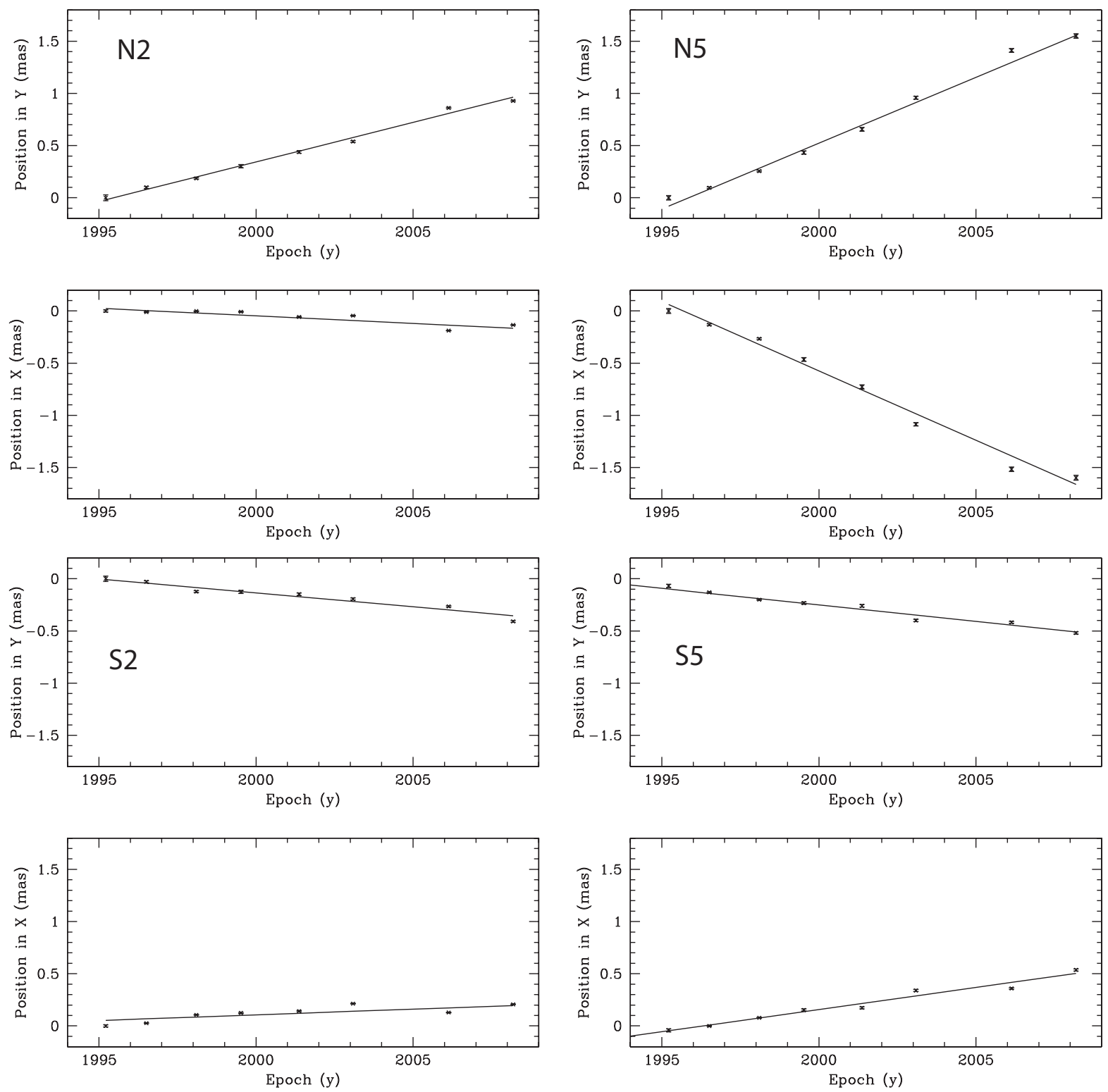

Fig. 5.- Velocities of N2, S2, N5 and S5 derived by fitting to the $\mathrm{X}$ and Y components of motion observed at $8.4 \mathrm{GHz}$. Velocities are tabulated in table 2. The zero point is taken to be the position of the component at the first epoch. 

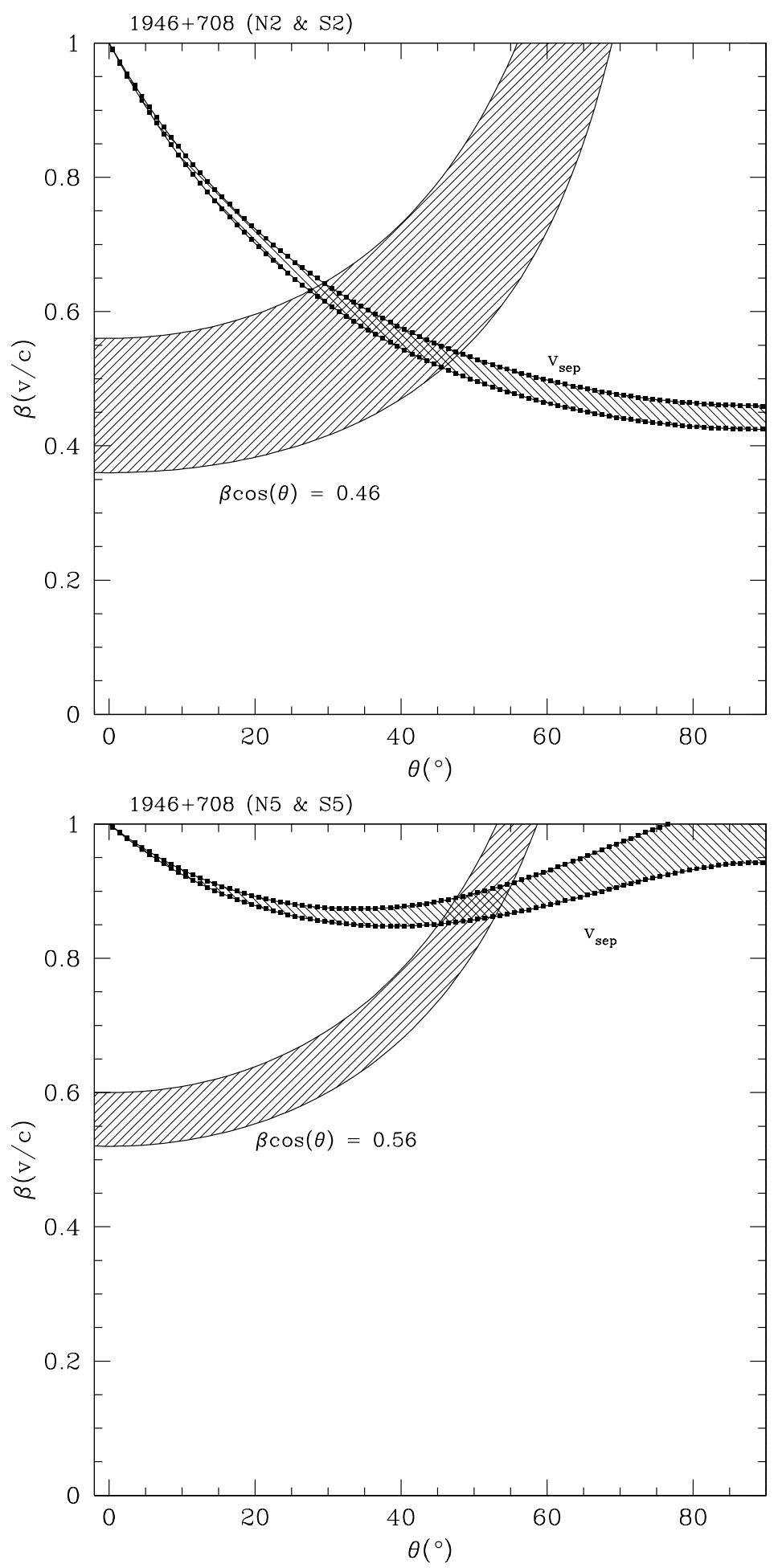

Fig. 6. - The intrinsic jet velocity $(\beta)$ plotted against the inclination of the source $(\theta)$ measured from the line-of-sight to the jet axis. The solid lines represent the constraint $\beta \cos \theta=0.46 \pm 0.1$ from the apparent velocity ratio $\mu_{a} / \mu_{r}=2.76 \pm 0.69$ of components $\mathrm{N} 2$ and S2 (top). The heavy dashed lines show the constraint from the apparent separation velocity $v_{s e p}=0.63 \pm 0.01 \mathrm{c}$ for $\mathrm{N} 2$ and $\mathrm{S} 2$ with $H_{0}=71 \mathrm{~km} \mathrm{~s}^{-1} \mathrm{Mpc}^{-1}$ assumed. At bottom is the same plot derived from apparent velocity measurements of the N5 and S5 pair $\left(\mu_{a} / \mu_{r}=3.50 \pm 0.44, \beta \cos \theta=0.56 \pm 0.04\right.$ and $\left.v_{\text {sep }}=1.40 \pm 0.01 \mathrm{c}\right)$ 

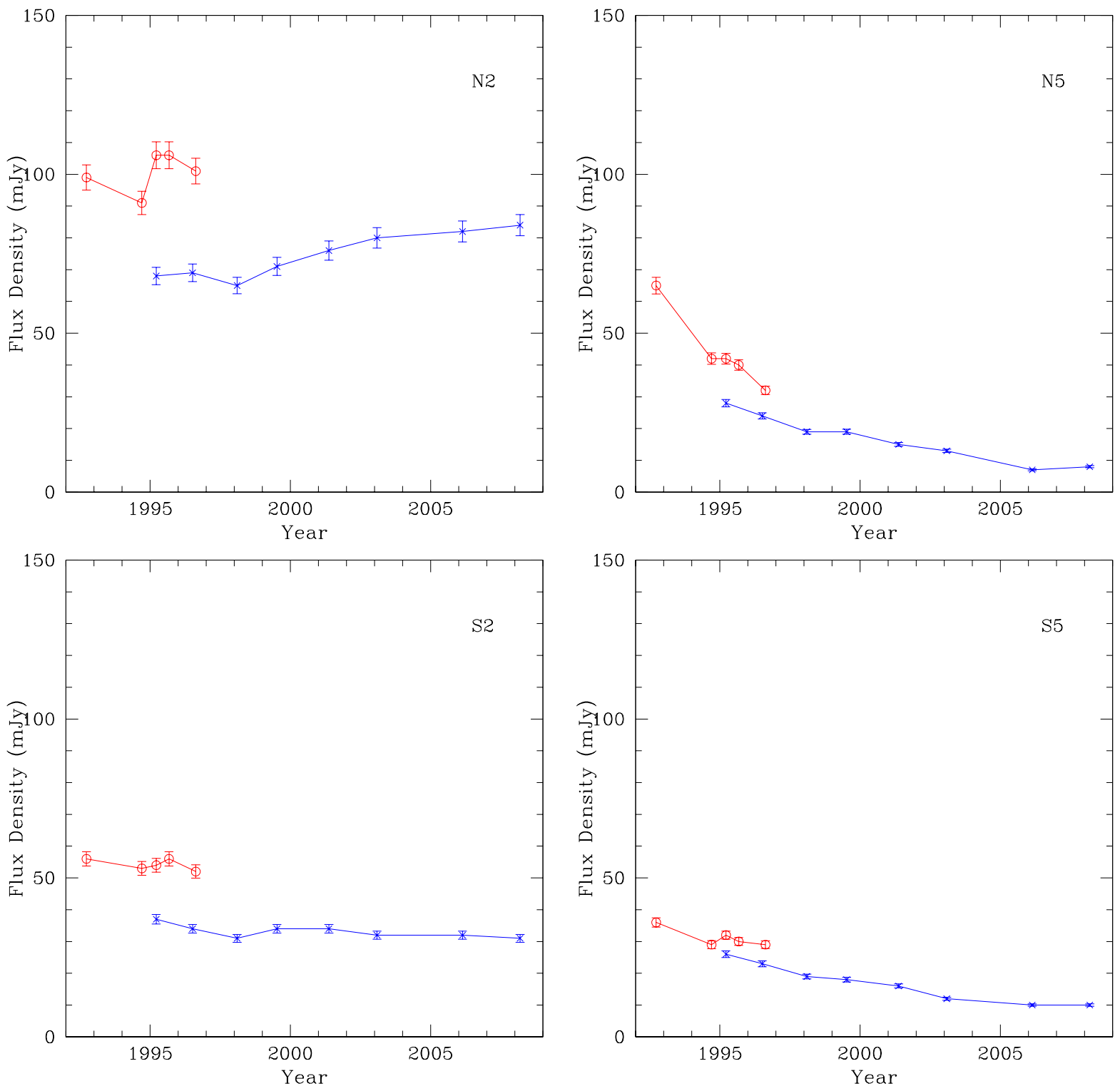

Fig. 7.- The flux densities at $5 \mathrm{GHz}$ (red), and $8.4 \mathrm{GHz}$ (blue), of the paired jet components N2/S2 and N5/S5 between 1992 and 2008 as derived from Gaussian modelfitting. 


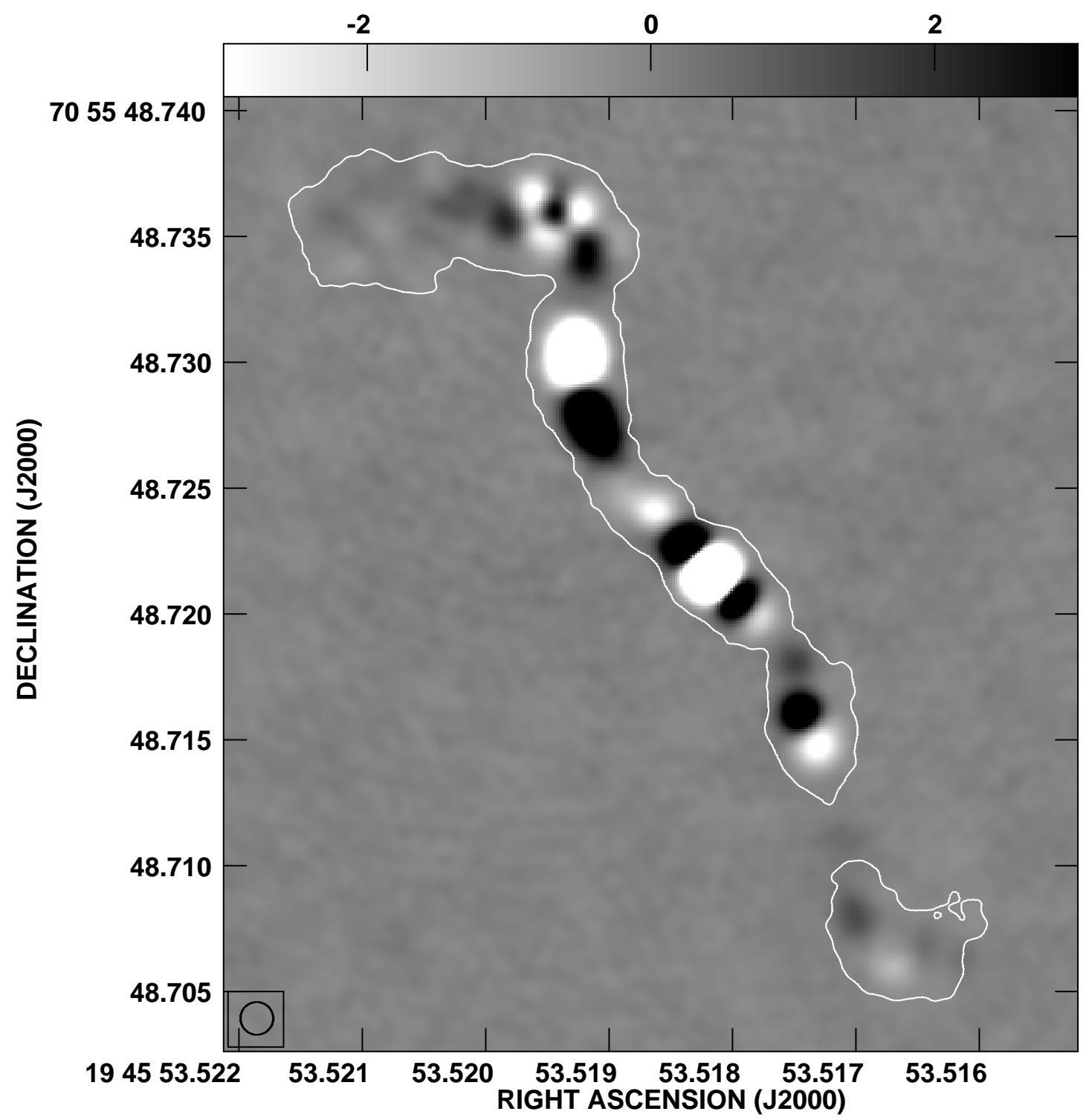

Fig. 8.- Difference image between the 1996 and 2008 epochs at 8.4 GHz. Both images were restored using a 1.3 mas circular beam, and the images were registered to the position of the northern hot spot using a two-dimensional gaussian fit implemented in the AIPS task JMFIT. The grey scale range is from -3 to $3 \mathrm{mJy} / \mathrm{beam}$, and the single contour level is from the 2008 epoch at $0.25 \mathrm{mJy} /$ beam. Lighter regions in indicate where the source was brighter in 2008 . 
Table 1. Observational Parameters FOR 1946+708

\begin{tabular}{rrrrrrr}
\hline \hline Date & $\begin{array}{r}\text { Frequency } \\
(\mathrm{GHz})\end{array}$ & $\begin{array}{r}\text { Bandwidth } \\
(\mathrm{MHz})\end{array}$ & $\begin{array}{r}\text { No.Ant } \\
\text { 1992 Sep 24 }\end{array}$ & $\begin{array}{r}\text { Scan Length } \\
(\mathrm{min})\end{array}$ & $\begin{array}{r}\text { Total Time } \\
(\text { hours })\end{array}$ & $\begin{array}{r}\text { rms } \\
(\mu \mathrm{Jy} / \mathrm{b})\end{array}$ \\
\hline 1994 Sep 15 & 5.0 & 2 & 15 & 20 & 0.92 & 340 \\
1995 Mar 22 & 5.0 & 16 & 12 & 20 & 0.95 & 588 \\
& 8.4 & 16 & 10 & 8 & 0.93 & 177 \\
& 15.4 & 16 & 10 & 2 & 3.83 & 163 \\
1995 Sep 3 & 5.0 & 8 & 10 & 8 & 2.13 & 315 \\
1996 Jul 7 & 8.4 & 32 & 9 & 6.5 & 0.98 & 235 \\
& 15.4 & 32 & 9 & 2 & 5.48 & 57 \\
1996 Aug 18 & 5.0 & 8 & 9 & 12 & 1.34 & 218 \\
1998 Feb 6 & 8.4 & 32 & 10 & 5.5 & 0.73 & 251 \\
& 15.4 & 32 & 10 & 2 & 4.67 & 45 \\
1999 Jul 11 & 8.4 & 32 & 9 & 10 & 1.21 & 99 \\
& 15.4 & 32 & 8 & 2 & 2.74 & 87 \\
2001 May 17 & 8.4 & 32 & 10 & 10 & 0.78 & 161 \\
& 15.4 & 32 & 10 & 2 & 3.49 & 76 \\
2003 Feb 2 & 8.4 & 32 & 14 & 10 & 1.74 & 250 \\
2006 Feb 18 & 8.4 & 32 & 14 & 4 & 6.67 & 55 \\
2008 Mar 9 & 8.4 & 32 & 14 & 4 & 3.33 & 38 \\
\hline
\end{tabular}

${ }^{1} 2003$ Feb 2 is the mean epoch for observations taken with the VLBA alone on January 24, 2003 and with a Global array consisting of the VLBA, Westerbork phased array, Onsala, Medicina and Noto on 2003 February 10. The observations were combined for calibration and imaging.

Table 2. Component Relative ${ }^{1}$ Motion Fitting Results

\begin{tabular}{lccccccc}
\hline \hline Component & $\begin{array}{c}\text { Velocity in } y \\
(\mathrm{mas} / \mathrm{y})\end{array}$ & $\chi^{2}$ & $\begin{array}{c}\text { Velocity in } x \\
(\mathrm{mas} / \mathrm{y})\end{array}$ & $\chi^{2}$ & $\begin{array}{c}\text { Velocity } \\
(\mathrm{mas} / \mathrm{y})\end{array}$ & $\begin{array}{c}\text { Velocity } \\
(\mathrm{c})\end{array}$ & $\begin{array}{c}\text { Angle of Motion } \\
\left({ }^{o}\right)\end{array}$ \\
\hline core & Reference Component & $\ldots$ & $\ldots$ & $\ldots$ & $\ldots$ & $\ldots$ & $\ldots$ \\
N2 & $0.076 \pm 0.0008$ & 0.90 & $-0.014 \pm 0.0002$ & 3.0 & $0.077 \pm 0.0008$ & $0.461 \pm 0.005$ & 10.4 \\
S2 & $-0.026 \pm 0.0008$ & 0.80 & $0.011 \pm 0.0004$ & 3.0 & $0.028 \pm 0.0009$ & $0.167 \pm 0.005$ & -157.1 \\
N5 & $0.126 \pm 0.0013$ & 1.4 & $-0.132 \pm 0.0014$ & 2.3 & $0.182 \pm 0.0019$ & $1.088 \pm 0.011$ & 46.3 \\
S5 & $-0.031 \pm 0.0008$ & 0.53 & $0.042 \pm 0.0007$ & 2.1 & $0.052 \pm 0.001$ & $0.311 \pm 0.006$ & -126.4 \\
\hline
\end{tabular}

${ }^{1}$ Errors quoted are for apparent motions relative to the core only, and do not include any motion of the core component. The systematic error that would be produced by motion of the core is estimated by the slow apparent relative motions of the northern hot spot (NHS) at $<0.005$ mas/yr in both Right Ascension and Declination. Only the $8.4 \mathrm{GHz}$ modelfit results are used.

${ }^{2}$ Angles measured from north through east. 